\title{
Surgical management of incidentally discovered low-grade gliomas
}

\author{
*Andrew J. Gogos, MBBS, FRACS,, Jacob S. Young, MD, ${ }^{1}$ Matheus P. Pereira, BS, ${ }^{2}$ \\ Ramin A. Morshed, MD, ${ }^{1}$ Matthew B. Potts, MD, ${ }^{3}$ Shawn L. Hervey-Jumper, MD, ${ }^{1}$ \\ and Mitchel S. Berger, MD ${ }^{1}$
}

\begin{abstract}
${ }^{1}$ Department of Neurological Surgery, University of California, San Francisco; ${ }^{2}$ School of Medicine, University of California, San Francisco, California; and ${ }^{3}$ Department of Neurological Surgery, Northwestern Memorial Hospital, Northwestern University Feinberg School of Medicine, Chicago, Illinois
\end{abstract}

OBJECTIVE Although most patients with low-grade glioma (LGG) present after a seizure, a small proportion is diagnosed after neuroimaging is performed for a sign or symptom unrelated to the tumor. While these tumors invariably grow, some surgeons argue for a watchful waiting approach. Here, the authors report on their experience in the surgical treatment of patients with incidental LGG (iLGG) and describe the neurological outcomes, survival, and complications.

METHODS Relevant cases were identified from a prospective registry of patients undergoing glioma resection at the University of California, San Francisco, between 1997 and 2019. Cases were considered iLGG when the lesion was noted on imaging performed for a reason unrelated to the tumor. Demographic, clinical, pathological, and imaging data were extracted from the electronic medical record. Tumor volumes, growth, and extent of resection were calculated from pre- and postoperative volumetric FLAIR sequences.

RESULTS One hundred thirteen of 657 (17.2\%) first-time resections for LGG were for incidental lesions. The most common reasons for the discovery of an iLGG were headaches (without mass effect, $34.5 \%$ ) or trauma (16.8\%). Incidental tumors were no different from symptomatic lesions in terms of laterality or location, but they were significantly smaller ( $22.5 \mathrm{vs} 57.5 \mathrm{~cm}^{3}, p<0.0001$ ). There was no difference in diagnosis between patients with iLGG and those with symptomatic LGG (sLGG), incorporating both molecular and pathological data. The median preoperative observation time for iLGG was 3.1 months (range 1 month-12 years), and there was a median growth rate of $3.9 \mathrm{~cm}^{3} / y e a r$. Complete resection of the FLAIR abnormality was achieved in $57 \%$ of patients with incidental lesions but only $23.8 \%$ of symptomatic lesions $(p<0.001)$, and the residual volumes were smaller for iLGGs $\left(2.9\right.$ vs $\left.13.5 \mathrm{~cm}^{3}, p<0.0001\right)$. Overall survival was significantly longer for patients with incidental tumors (median survival not reached for patients with iLGG vs 14.6 years for those with sLGG, $p$ <.0001). There was a 4.4\% rate of neurological deficits at 6 months.

CONCLUSIONS The authors present the largest cohort of iLGGs. Patient age, tumor location, and molecular genetics were not different between iLGGs and sLGGs. Incidental tumors were smaller, a greater extent of resection could be achieved, and overall survival was improved compared to those for patients with sLGG. Operative morbidity and rates of neurological deficit were acceptably low; thus, the authors advocate upfront surgical intervention aimed at maximal safe resection for these incidentally discovered lesions.

https://thejns.org/doi/abs/10.3171/2020.6.JNS201296

KEYWORDS incidental glioma; symptomatic glioma; molecular genetics; extent of resection; malignant transformation; survival; oncology

A LTHOUGH most patients with low-grade glioma (LGG) present after a seizure, ${ }^{1,2}$ a small proportion presents after imaging is performed for a sign or symptom unrelated to the tumor. Once incidental, asymptomatic lesions are found, some argue for the safety of a watchful waiting approach, in which surgical intervention is deferred until the patient experiences symptoms or has imaging findings suggestive of growth or high-grade features. ${ }^{3,4}$ However, studies have demonstrated that observation of incidental LGG (iLGG) is invariably associated

ABBREVIATIONS EOR = extent of resection; GBM = glioblastoma multiforme; iLGG = incidental LGG; LGG = low-grade glioma; $\mathrm{sLGG}=$ symptomatic LGG; UCSF = University of California, San Francisco.

SUBMITTED April 16, 2020. ACCEPTED June 1, 2020.

INCLUDE WHEN CITING Published online October 2, 2020; DOI: 10.3171/2020.6.JNS201296.

${ }^{*}$ A.J.G. and J.S.Y. contributed equally to this work. 
with tumor growth, with a yearly growth rate of around 4 $\mathrm{mm} \cdot{ }^{5-7}$ Further, the approach of watchful waiting for LGG has been associated with a worse overall survival and a higher risk of malignant transformation. ${ }^{8}$

We and others have shown that early surgical intervention for iLGG is associated with a better extent of resection (EOR) and improved survival ${ }^{6,9-12}$ perhaps because incidental lesions are usually smaller and located in noneloquent areas. ${ }^{9}$ Further, improvements in mapping and monitoring techniques have meant that even incidental lesions within eloquent regions can often be safely resected.13

Here, we report on our experience of treating patients with iLGG via resection and describe the neurological outcomes, survival, and complications.

\section{Methods}

\section{Patient Identification and Clinical Data}

Relevant cases were identified from a prospective registry of patients undergoing grade II glioma resection at the University of California, San Francisco (UCSF) between 1997 and 2019. The indication for neuroimaging was reviewed for all cases of grade II diffuse gliomas. Cases were considered "incidental" when the lesion was noted on imaging performed for a reason unrelated to the tumor, including research participation, trauma, headache (note that headaches were only considered unrelated to the tumor in patients with tumors without mass effect), sinus symptoms, or neurological symptoms not related to the tumor (e.g., hearing loss or ipsilateral limb sensory changes). Demographic, clinical, pathological, and imaging data were retrospectively extracted from the electronic medical record. New neurological deficits after surgery were considered permanent if they persisted for more than 6 months, except in one patient whose supplemental motor area syndrome resolved completely after 9 months. Molecular subtyping was available for most cases. Patients were only considered to have undergone malignant transformation if such was confirmed histologically during repeat resection. IRB approval was granted by UCSF.

Resection was recommended for all patients with imaging findings thought to represent LGG. For patients with equivocal lesions, serial imaging was performed, and resection was recommended if the lesions grew. In the majority of cases, serial imaging had been performed externally prior to referral. Histopathological specimens were obtained intraoperatively, during tumor removal. Resection was completed using awake mapping and monitoring when eloquent areas were believed to be at risk by the senior author, with the techniques described elsewhere. ${ }^{14,15}$ If only motor mapping was necessary, then asleep motor mapping was performed in most cases. ${ }^{16}$ Intraoperative MRI was not used during surgical resection for these patients. The goal of resection was to remove as much of the FLAIR abnormality as safely possible.

\section{Imaging Analysis}

Tumor volumes and EOR were calculated from pre- and postoperative volumetric FLAIR sequences by using the Brainlab Elements SmartBrush tool (version 2.6) or, for patients included in a previously published series (updated data presented here), custom in-house software written in $\mathrm{C}$ and Matlab. ${ }^{9}$ We also calculated tumor volumes for patients who had one or more MRI scans available at least 6 months prior to the date of operation by using available FLAIR sequences to assess for the rate of tumor growth.

\section{Statistical Analysis}

A Student t-test or the Mann-Whitney U-test was used when comparing parametric and nonparametric continuous variables, as appropriate. The chi-square test and Fisher exact test were used for categorical variables. Survival analysis was performed using the Kaplan-Meier approach with the Cox proportional hazards model. All statistical analyses were performed in $\mathrm{R}$ version 3.5.3 (R Foundation for Statistical Computing), and a $\mathrm{p}$ value less than 0.05 was considered statistically significant.

\section{Results}

From February 1998 to August 2019, 685 first-time resections for LGG were performed, and the records for $657(95.9 \%)$ patients were available. One hundred thirteen of the $657(17.2 \%)$ cases were incidental, whereas $544(82.8 \%)$ cases presented with symptoms referable to the lesions. Thirty-five iLGG cases have been reported on previously, although updated data are presented here. ${ }^{9}$ Median follow-up was 6.6 years for iLGGs (IQR 3.5-21.1 years) and 6.1 years for symptomatic LGGs (sLGGs; IQR 2.5-19.5 years; $\mathrm{p}=0.19$ ). Over time, iLGGs became a higher proportion of cases performed at UCSF, from $8.5 \%$ of all first resections for LGG in the first 3 years of this series to $23 \%$ in the last 2 years (Fig. 1).

The most common reasons for the discovery of incidental lesions were headaches $(34.5 \%)$ or trauma $(16.8 \%$; Table 1). Typically, the headaches were described as "migraine" or "episodic" and occurred in tumor cases without mass effect or midline shift. Headaches were more common in women than men $(42.9 \%$ vs $24.0 \%, p=0.047)$, whereas the converse was true for trauma $(7.9 \%$ in women vs $28.0 \%$ in men, $p=0.006$ ). Seizures were the most common presenting symptoms for patients with sLGG (88.8\%), with no difference between women and men $(87.0 \%$ vs $90.0 \%, p=0.50)$. Patients with iLGG were more likely to be female ( $p=0.002)$ but, importantly, were of similar age to patients with sLGG. Incidental tumors were no different from sLGGs in terms of laterality or location, although they were significantly smaller $\left(22.5 \mathrm{vs} 57.5 \mathrm{~cm}^{3}\right.$, $\mathrm{p}<0.0001$ ) than symptomatic tumors.

In 43 of 113 patients with iLGG, the tumor was observed with serial imaging for more than 6 months prior to resection (with or without biopsy). The median preoperative observation time was 3.1 months (range 1 month-12 years). All observed tumors grew, with a median growth rate of $3.9 \mathrm{~cm}^{3}$ per year. Figure 2 depicts the relative and absolute volumetric growth of the serially imaged tumors.

There was no difference in the molecular and pathological diagnoses between patients with iLGG and those with sLGG (Table 2). Slightly more than half of the iLGGs and the sLGGs were 1p/19q co-deleted lesions (i.e., oligodendrogliomas), and approximately $10 \%$ of lesions were $I D H$-wildtype, which most groups would consider to be 


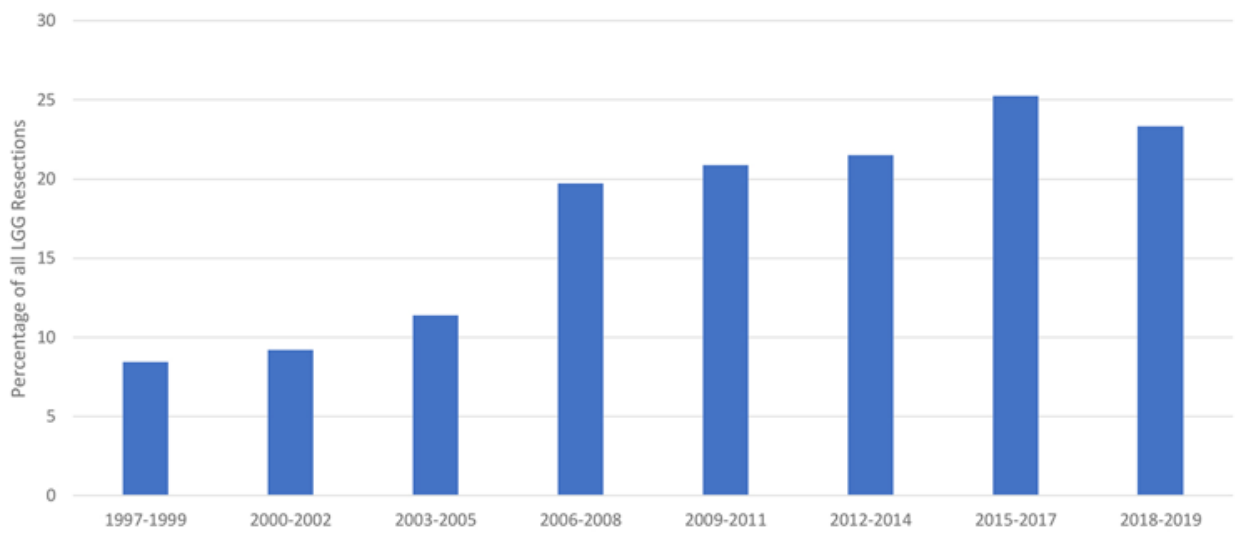

FIG. 1. Proportions of LGG resections performed at UCSF that were for incidental lesions. Over time, there was an increase in the rate of iLGGs. Figure is available in color online only.

TABLE 1. Summary of characteristics of patients with ILGG and SLGG

\begin{tabular}{|c|c|c|c|}
\hline Variable & iLGG (n = 113) & sLGG $(n=544)$ & $p$ Value \\
\hline \multicolumn{4}{|l|}{ Sex, no. (\%) } \\
\hline Female & $63(55.8)$ & $215(39.5)$ & \multirow{2}{*}{0.002} \\
\hline Male & $50(44.2)$ & $327(60.1)$ & \\
\hline Mean age in yrs (range) & $39.4(18.9-71.3)$ & $39.8(19.2-76.7)$ & 0.78 \\
\hline Reason for initial imaging, no. (\%) & & & NA \\
\hline Headache & $39(34.5)^{*}$ & $33(6.1)$ & \\
\hline Seizures & & $483(88.8)$ & \\
\hline Trauma & $19(16.8)$ & & \\
\hline Nonlocalized neurology & $17(15.0)$ & & \\
\hline Otorhinolaryngology disorder & $15(13.3)$ & & \\
\hline Research participant & $4(3.5)$ & & \\
\hline Focal symptoms & & $28(5.1)$ & \\
\hline Other† & $19(16.8)$ & & \\
\hline \multicolumn{4}{|l|}{ Tumor side, no. (\%) } \\
\hline Lt & $67(59.3)$ & $308(56.6)$ & \multirow{3}{*}{0.75} \\
\hline $\mathrm{Rt}$ & $46(40.7)$ & $231(42.5)$ & \\
\hline Bilat & $0(0.0)$ & $5(0.9)$ & \\
\hline \multicolumn{4}{|l|}{ Tumor site, no. (\%) } \\
\hline Frontal & $66(58.4)$ & $273(50.2)$ & \multirow{7}{*}{0.58} \\
\hline Temporal & $16(14.2)$ & $88(16.2)$ & \\
\hline Insula & $15(13.3)$ & $123(22.6)$ & \\
\hline Parietal & $10(8.8)$ & $52(9.6)$ & \\
\hline Cingulate & $2(1.8)$ & $4(0.7)$ & \\
\hline Occipital & $1(0.9)$ & $0(0)$ & \\
\hline Multiple & $3(2.7)$ & $4(0.7)$ & \\
\hline Mean tumor vol in $\mathrm{cm}^{3}$ & 22.5 & 57.5 & $<0.0001$ \\
\hline Median observation time in mos $(n=43)$ & $3.1(1-144)$ & NA & \\
\hline Median growth during observation in $\mathrm{cm}^{3} / \mathrm{yr}(\mathrm{n}=43)$ & 3.9 & NA & \\
\hline
\end{tabular}

$\mathrm{n}=$ number of cases; $\mathrm{NA}=$ not available.

Boldface type indicates statistical significance.

* Includes migraine, tension-type headaches, muscle-contraction headaches, cluster headaches, and sinusitis. Patients with signs suggestive of raised intracranial pressure or mass effect on MRI were excluded.

† Other = screening imaging in the context of other disease (i.e., hematological malignancies, sarcoid, hypopituitarism or other symptomatic brain tumor). 

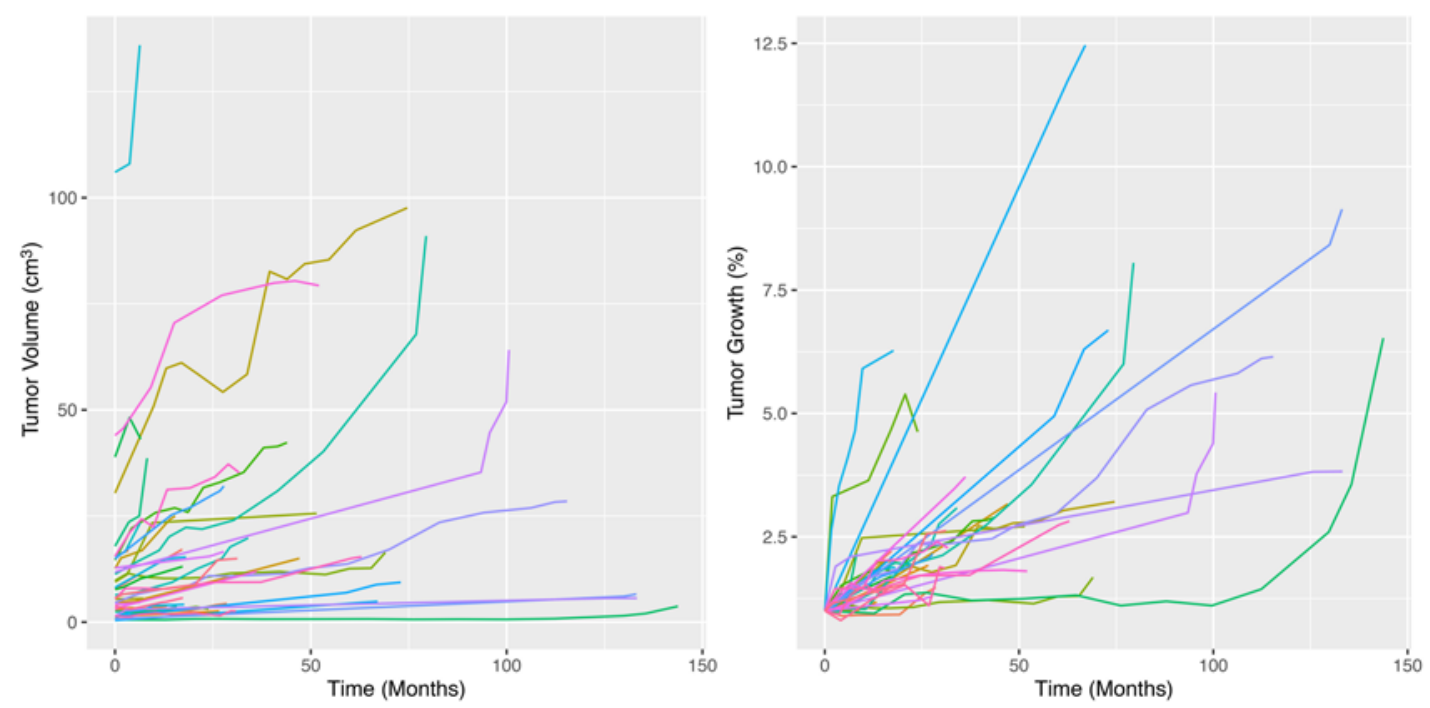

FIG. 2. Left: Absolute volumetric growth of observed iLGGs. Plot characterizing the change in volume ( $\left.\mathrm{cm}^{3}\right)$ over time (months). Each line represents the rate of iLGG growth for an individual patient $(n=43)$ during the period of observation prior to surgical intervention. All tumors were seen to change in size, albeit with different rates of change. Some patients demonstrated an acceleration in the rate of change immediately prior to surgical intervention. Right: Relative growth of observed iLGGs. Plot characterizing the percent of tumor growth over time (months). Each line represents the percent change in iLGG size for an individual patient $(n=$ 43) during the period of observation prior to surgical intervention. Again, all tumors were observed to grow prior to intervention.

molecular glioblastoma multiforme (GBM) despite their low grade. Complete resection was achieved in $57.1 \%$ of patients with incidental lesions but only $23.8 \%$ of symptomatic lesions $(\mathrm{p}<0.001)$, and the residual volumes were smaller for iLGGs ( 2.9 vs $\left.13.5 \mathrm{~cm}^{3}, \mathrm{p}<0.0001\right)$.

The rates of complications for patients with iLGG are shown in Table 3. Three (2.7\%) patients had medical complications. One patient suffered Stevens-Johnson syndrome from phenytoin use after levetiracetam had failed to control postoperative seizures. One patient had a nondesquamating phenytoin rash, and one patient had ileus that was managed conservatively. Five (4.4\%) patients had minor surgical complications, which included 3 postoperative seizures managed with antiepileptic medication, and 2 patients developed pseudomeningoceles, neither of whom required operative intervention.

Postoperative neurological deficits are also listed in Table 3, and for the majority of immediate deficits the patients obtained a full recovery. One patient had a permanent Medical Research Council (MRC) grade 3/5 hemiplegia after resection of a tumor within the primary motor cortex. The other motor deficit was a mild facial droop in an otherwise intact patient. The permanent speech deficit was a patient with intermittent paraphasic errors. The two permanent visual deficits were a hemianopia and quadrantanopia. Five patients with initial neurological deficits were lost to follow-up prior to 6 months.

Overall survival was significantly longer for patients with incidental tumors than for those with sLGG (Fig. 3A). Median survival for patients with sLGG was 14.6 years, whereas the median survival for patients with iLGG was not reached during the study period (HR $0.24,95 \%$ CI $0.11-0.51$, p < $0.0001, \log$-rank test). We controlled for EOR by only look- ing at patients with an EOR $\geq 95 \%$, and even in this subgroup, iLLG was still associated with significantly longer overall survival ( $\mathrm{p}=0.032$, log-rank test; Fig. 3B).

Twenty-seven (23.9\%) patients with iLGG received postoperative chemotherapy, and 9 of them were also treated with radiotherapy. Adjuvant treatment was administered most commonly after radiological progression. Twenty-seven $(23.9 \%)$ patients had a subsequent resection, and in $11(9.7 \%)$ patients malignant transformation was demonstrated (3 anaplastic astrocytoma, 2 anaplastic oligodendroglioma, and $5 \mathrm{IDH}$-mutated GBM and $1 \mathrm{IDH}$ wildtype GBM). Moreover, $36.4 \%$ of patients with malignant transformation had been treated with chemotherapy prior to transformation, compared with $22.5 \%$ of patients without transformation. However, this difference was not statistically significant $(p=0.29)$. Although the rate of malignant transformation was higher in the symptomatic group (20.2\% in sLGG vs $9.7 \%$ in iLGG), there was no difference in the rate of malignant transformation using survival analysis (Fig. 3C).

\section{Discussion}

Although some groups have suggested active surveillance of iLGG, others advocate for early surgical intervention..$^{9,17,18}$ However, biopsy and watchful waiting have been shown to be inferior to early resection in cohorts of both iLGGs and sLGGs. ${ }^{8,19}$ Here, we expand on a previously published series from our institution for patients who undergo surgery for iLGG. ${ }^{9}$ We found that the proportion of LGGs treated with resection at our institution has been increasing over time, with iLGGs making up between $20 \%$ and $25 \%$ of low-grade tumors treated with resection. Although some of this increase is likely the re- 
TABLE 2. Pathological diagnosis, EOR, and survival for patients with newly diagnosed LGG

\begin{tabular}{lccc}
\hline \multicolumn{1}{c}{ Variable } & iLGG & sLGG & p Value \\
\hline Molecular classification & $\mathrm{n}=85$ & $\mathrm{n}=342$ & \\
IDH-wildtype & $11(12.9)$ & $32(9.4)$ & \\
IDH-mutated, 1p19q intact & $26(30.6)$ & $99(28.9)$ & 0.31 \\
IDH-mutated, 1p19q co-deleted & $47(55.3)$ & $196(57.3)$ & \\
Other 1p19q status & $1(1.2)$ & $15(4.4)$ & \\
\hline EOR & $\mathrm{n}=112$ & $\mathrm{n}=411$ & \\
Median volumetric EOR (IQR) & $100 \%(90.4-100 \%)$ & $87.45 \%(71.4-100 \%)$ & $<0.001$ \\
100\% & $64(57.1)$ & $98(23.8)$ & $<0.001$ \\
$\geq 95 \%$ & $77(68.8)$ & $146(35.5)$ & 0.024 \\
$\geq 90 \%$ & $86(76.8)$ & $191(46.5)$ & 0.001 \\
$\geq 80 \%$ & $95(84.8)$ & $258(62.8)$ & $<0.0001$ \\
$\geq 70 \%$ & $101(90.2)$ & $313(76.2)$ & $<0.0001$ \\
\hline Mean residual vol in cm ${ }^{3}$ & 2.9 & 13.5 & $<0.0001$ \\
\hline Median survival in yrs & Not reached & 14.6 & $<0.0001$ \\
\hline FU in yrs & & & \\
Median & 6.6 & 6.1 & \\
Mean & 7.5 & 6.8 & \\
IQR & $3.5-21.1$ & $2.5-19.5$ & \\
\hline Median KPS score (range) & & & \\
Preop & $100\left(50^{*}-100\right)$ & & \\
Postop & $90\left(50^{*}-100\right)$ & & \\
\hline
\end{tabular}

$\mathrm{FU}=$ follow-up; KPS $=$ Karnofsky Performance Status.

Values expressed as number (\%), unless indicated otherwise. Boldface type indicates statistical significance.

${ }^{*}$ Patient wheelchair bound from perinatal spinal cord injury.

sult of referral patterns, the increased ease and prevalence of neuroimaging also likely contributed to the rise in cases. ${ }^{20-22}$ MRI utilization has increased fourfold in the US and Canada, going from 16 cases per 1000 person-years in 2000 to 64 cases per 1000 person-years in $2016,{ }^{23}$ and we suspect iLGG will be encountered more frequently in the future by all neurosurgical oncologists. Given our findings, we believe these tumors can be safely removed and patients can benefit from early surgical intervention.

TABLE 3. Perioperative complications in 113 patients with iLGG

\begin{tabular}{lccc}
\hline \multicolumn{1}{c}{ Complication } & No. (\%) & $\begin{array}{c}\text { Permanent Deficit } \\
\text { (no. [\%] })^{*}\end{array}$ & $\begin{array}{c}\text { Lost to FU } \\
\text { (no. [\%]) }\end{array}$ \\
\hline Any medical complication & $3(2.7)$ & & \\
\hline Surgical complication & $5(4.4)$ & & \\
\hline $\begin{array}{l}\text { New neurological deficit } \\
\text { immediately postop }\end{array}$ & & & \\
$\quad$ SMA syndrome & $5(4.4)$ & $0(0.0)$ & $1(0.9)$ \\
$\quad$ Language & $9(8.0)$ & $1(0.9)$ & $1(0.9)$ \\
$\quad$ Motor & $6(5.3)$ & $2(1.8)$ & $1(0.9)$ \\
$\quad$ Visual & $4(3.5)$ & $2(1.8)$ & $1(0.9)$ \\
$\quad$ Gerstmann's syndrome & $1(0.9)$ & $0(0.0)$ & $1(0.9)$ \\
\hline
\end{tabular}

SMA = supplementary motor area.

* The patient with a language deficit had speech described as nonfluent with paraphasic errors. Regarding motor deficits, 1 patient had Medical Research Council (MRC) grade $3 / 5$ weakness (but was ambulant) and another had a slight facial droop. The 2 permanent visual deficits were a homonymous hemianopia and a homonymous quadrant loss.
Many patients have a period of observation prior to resection, frequently at outside institutions. We found that all observed iLGGs grew, with a median growth rate of $3.9 \mathrm{~cm}^{3} /$ year. This is consistent with rates published by other groups. ${ }^{5,6,9}$ Interestingly, for lesions that were followed with serial imaging, different rates of growth were observed. Future work is needed to determine if tumor growth rates are related to the tumor molecular profile and to define the importance that this interval growth has on patient outcomes such as progression-free survival and overall survival. Nevertheless, given that these lesions invariably grow, there appears to be no utility in waiting for symptoms to arise before intervening surgically.

We found that overall survival was longer for patients with iLGG than for those with sLGG. Although this finding may be related to the superior EOR in the iLGG group, we found increased survival when looking at the subset of patients with $\geq 95 \%$ EOR. This may relate to malignant transformation. Malignant transformation has typically been reported in approximately $20 \%$ of LGGs, which is approximately double the rate in our series.24 Moreover, risk factors for malignant transformation include treatment with chemotherapy alone and amount of residual disease. iLGGs can frequently be completely (or nearly completely) resected and, as a result, may not require adjuvant chemotherapy. Therefore, there is a plausible biological mechanism underlying the finding that early surgical treatment of iLGG can reduce the risk of malignant transformation. Importantly, neurooncologists often consider LGGs low risk if the patient is younger than 40 years of age and undergoes complete resection of the 

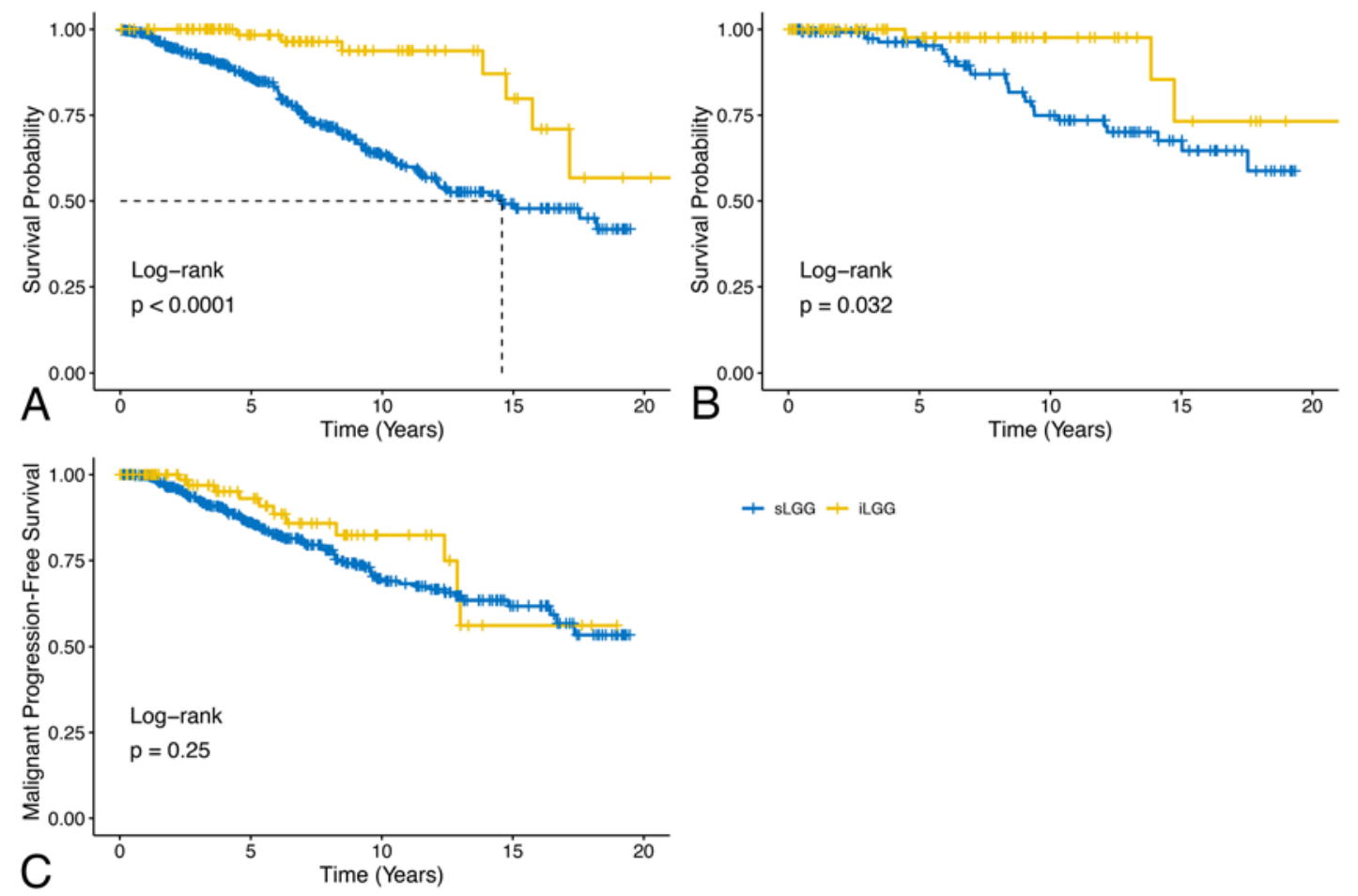

$+\mathrm{sLGG}+\mathrm{iLGG}$

FIG. 3. A: Overall survival in patients with iLGG and sLGG. Median survival for patients with sLGG was 14.6 years, whereas median survival for patients with iLGG was not reached during the study period $(p<0.0001)$. B: Overall survival in patients with EOR $\geq 95 \%$. iLLG was still associated with significantly longer overall survival than that for patients with sLGG even for those in whom an EOR $\geq 95 \%$ was achieved ( $p=0.032$ ). C: Malignant progression-free survival. Although the rate of malignant transformation was higher in the symptomatic group $(20.2 \%$ in sLGG vs $9.7 \%$ in iLGG), there was no difference in the rate of malignant transformation using survival analysis $(p=0.25)$.

FLAIR abnormality. ${ }^{25}$ Adjuvant therapy is typically withheld in low-grade, low-risk patients. Therefore, early surgery for iLGG may avoid the side effects, complications, and added healthcare costs associated with adjuvant treatment. Of equal importance, $13 \%$ of the iLGGs were found to be $I D H$-wildtype. Since this cohort has been shown to have worse outcomes, these patients likely warrant more aggressive adjuvant therapy, which would not be known if these lesions were simply observed.

To our knowledge, this represents the largest series of iLGGs to date. A comparison with other published series can be found in Table 4. Although we found a moderate rate of transient deficits, such is not unexpected when considering that our cohort consisted of lesions frequently in or near eloquent regions. Moreover, in contrast to other groups, a greater percentage of lesions in our study was left-sided. Additionally, our median EOR of $100 \%$ was superior to most EORs in other published cohorts. Although permanent deficits occurred in $4.4 \%$ of patients with iLGG, most of them were not of major functional significance. This rate is similar to those reported in other series of patients with LGG. ${ }^{2,26-28}$ The rates of neurological deficit reported in this series may not be generalizable to all centers. For lesions in eloquent or high-risk areas, such as the insula, optimal outcomes are likely found at highvolume neurosurgical oncology centers. ${ }^{29}$

Interestingly, some authors argue that patients with
iLGG actually have neuropsychological changes, even though they have intact neurological examinations. ${ }^{30}$ While it may be the case that some iLGGs are not truly asymptomatic, since neuropsychological assessment is typically not a standard evaluation in the workup of patients with LGG, we do not believe the presence of symptoms or signs should influence how these lesions are managed. Moreover, the interaction between glioma cells and neuronal cells is poorly understood, ${ }^{31,32}$ and the threshold that must be passed for a tumor to become symptomatic is unknown. It is possible that tumors with certain genetic profiles are more poorly "tolerated" by neuronal tissue and cause symptoms quicker than other tumors, independent of location, which may be a prognostic factor in and of itself. This exciting area of glioma-neuronal cell interaction will be an important area of future investigation. Finally, another factor for surgeons to consider when deciding whether or not to treat an incidental lesion is the anxiety created by the knowledge that the brain lesion is only being monitored and not treated. Future work should focus on the quality of life changes associated with treating these lesions early after their discovery.

For this study, we considered headaches to be symptomatic presentations of LGG when there was imaging evidence or headache descriptors consistent with raised intracranial pressure or when there was mass effect from the tumor on local structures (Fig. 4). However, headache 
TABLE 4. Literature survey of iLGG series

\begin{tabular}{|c|c|c|c|c|c|c|c|c|c|}
\hline Authors \& Year & $\begin{array}{l}\text { Total No. } \\
(\%) \text { of Cases } \\
\text { Over Study } \\
\text { Period }\end{array}$ & $\begin{array}{l}\text { Median } \\
\text { Size } \\
\left(\mathrm{cm}^{3}\right)\end{array}$ & $\begin{array}{l}\text { Median } \\
\text { Volumetric } \\
\text { EOR }\end{array}$ & $\begin{array}{c}\text { Included } \\
\text { Symptomatic } \\
\text { Comparison Group? } \\
\text { (no. of cases) }\end{array}$ & $\begin{array}{c}\text { Growth } \\
\text { During } \\
\text { Observation }\end{array}$ & Left-Sided & $\begin{array}{l}\text { Neurological } \\
\text { Deficit }\end{array}$ & $\begin{array}{l}\text { Median } \\
\text { FU } \\
\text { (yrs) }\end{array}$ & $\begin{array}{l}\text { No. of Malignant } \\
\text { Transformation } \\
\text { Cases (\%) }\end{array}$ \\
\hline $\begin{array}{l}\text { Pallud et al., } \\
2010^{6}\end{array}$ & $47(3.8)$ & 17.2 & NR & Yes, 1249 & $3.5 \mathrm{~mm} / \mathrm{yr}$ & $49 \%$ & NR & 6.6 & $\begin{array}{c}8 \text { in bx only patients } \\
(100), 6 \text { after } \\
\text { resection (15) }\end{array}$ \\
\hline Potts et al., $2012^{9}$ & $35(9.6)$ & 20.2 & $100 \%$ & Yes, 197 & $2.29 \mathrm{~cm}^{3} / \mathrm{yr}$ & $51.7 \%$ & 5 transient & $5.1^{*}$ & $4(11.4)$ \\
\hline Duffau, $2012^{13} \dagger$ & 11 & 32.6 & $100 \%$ & No & NR & $100 \%$ & $\begin{array}{l}7 \text { transient, } \\
0 \text { permanent }\end{array}$ & 1.7 & 0 \\
\hline Zhang et al., $2014^{5}$ & 23 & 23.8 & NR & Yes, 196 & NR & NR & NR & 9.3 & NR \\
\hline Lima et al., $2017^{12}$ & 19 & 51.4 & $100 \%$ & No & NR & $\ddagger$ & $\begin{array}{l}10 \text { transient, } \\
0 \text { permanent }\end{array}$ & 3.4 & NR \\
\hline $\begin{array}{l}\text { Opoku-Darko et } \\
\text { al., } 2018^{10}\end{array}$ & $34(6.8)$ & 57.2 & $84.5 \%$ & No & NR & $56 \%$ & $\begin{array}{l}1 \text { transient, } \\
1 \text { permanent }\end{array}$ & 5.1 & $5(14.7)$ \\
\hline lus et al., $2020^{11}$ & $34(10.2)$ & 15 & $100 \%$ & Yes, 223 & NR & $41.2 \%$ & $\begin{array}{c}3 \text { initial, } \\
0 \text { permanent }\end{array}$ & 5.8 & NR \\
\hline Present study§ & $113(17.2)$ & 22.5 & $100 \%$ & Yes, 544 & $3.9 \mathrm{~cm}^{3} / \mathrm{yr} \rrbracket$ & $59.3 \%$ & $\begin{array}{l}25 \text { initial, } \\
5 \text { permanent }\end{array}$ & 6.6 & $11(9.7)$ \\
\hline
\end{tabular}

bx = biopsy; NR = not reported.

${ }^{*}$ Mean value.

† This was a series of awake resections for iLGG.

$\ddagger$ All were described as eloquent, but the side was not indicated.

$\S$ Included the cases $(n=35)$ from Potts et al. ${ }^{9}$ but with expanded follow-up data. In $n=43$.

descriptors do have some subjectivity, and it is possible that there is some overlap between the two groups. Since headaches are one of the most common chief complaints in all of medicine and frequently lead to neuroimaging, we believe that attributing headaches to the presence of a tumor, when appropriate, is an important distinction for neurosurgeons to make, even though headache etiology is not always clear.

One limitation of this study is the lack of an observation-only control group. While we do not believe there is clinical equipoise in having such a cohort, we are unable to directly compare iLGGs that are only monitored with those that are resected. However, since most iLGGs will eventually become sLGGs, we believe that our comparison against sLGG is appropriate. Nevertheless, it is theoretically possible that some iLGGs may never go on to cause symptoms and move into the sLGG group.

\section{Conclusions}

We present the largest published series of iLGGs and include clinical, radiological, molecular, and outcome data. We demonstrated that incidental tumors are smaller than symptomatic lesions and are associated with a greater EOR. Incidental lesions were more common in women; however, patient age, tumor location, and molecular genetics were not different between iLGG and sLGG. Operative morbidity was low, and iLGG patient survival was greater than sLGG patient survival, even when comparing patients with an $\mathrm{EOR} \geq 95 \%$.
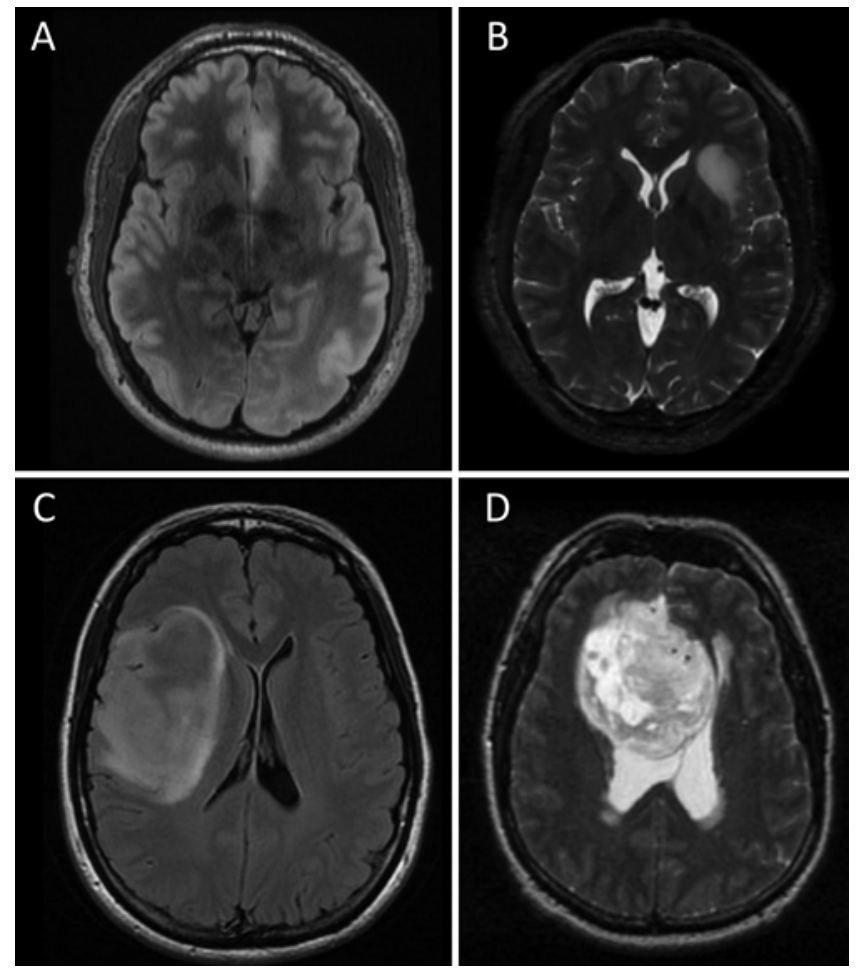

FIG. 4. T2 FLAIR (A) and T2-weighted (B) MRI sequences from two patients presenting with headaches revealed tumors without mass effect or midline shift and thus were considered to be incidental lesions. Compare those images to T2 FLAIR (C) and T2-weighted (D) MRI sequences from two patients presenting with headaches who were found to have large tumors with notable mass effect and thus were considered to be symptomatic lesions. 


\section{References}

1. Kurzwelly D, Herrlinger U, Simon M. Seizures in patients with low-grade gliomas-incidence, pathogenesis, surgical management, and pharmacotherapy. Adv Tech Stand Neurosurg. 2010;35:81-111.

2. Smith JS, Chang EF, Lamborn KR, et al. Role of extent of resection in the long-term outcome of low-grade hemispheric gliomas. J Clin Oncol. 2008;26(8):1338-1345.

3. Olson JD, Riedel E, DeAngelis LM. Long-term outcome of low-grade oligodendroglioma and mixed glioma. Neurology. 2000;54(7):1442-1448.

4. Recht LD, Lew R, Smith TW. Suspected low-grade glioma: is deferring treatment safe? Ann Neurol. 1992;31(4):431-436.

5. Zhang ZY, Chan AKY, Ng HK, et al. Surgically treated incidentally discovered low-grade gliomas are mostly IDH mutated and 1p19q co-deleted with favorable prognosis. Int $J$ Clin Exp Pathol. 2014;7(12):8627-8636.

6. Pallud J, Fontaine D, Duffau H, et al. Natural history of incidental World Health Organization grade II gliomas. Ann Neurol. 2010;68(5):727-733.

7. Noorani I, Sanai N. Surgical management of incidental gliomas. Neurosurg Clin N Am. 2017;28(3):397-406.

8. Jakola AS, Myrmel KS, Kloster R, et al. Comparison of a strategy favoring early surgical resection vs a strategy favoring watchful waiting in low-grade gliomas. JAMA. 2012; 308(18):1881-1888.

9. Potts MB, Smith JS, Molinaro AM, Berger MS. Natural history and surgical management of incidentally discovered low-grade gliomas. J Neurosurg. 2012;116(2):365-372.

10. Opoku-Darko M, Lang ST, Artindale J, et al. Surgical management of incidentally discovered diffusely infiltrating lowgrade glioma. J Neurosurg. 2018;129(1):19-26.

11. Ius $\mathrm{T}$, Cesselli $\mathrm{D}$, Isola $\mathrm{M}$, et al. Incidental low-grade gliomas: single-institution management based on clinical, surgical, and molecular data. Neurosurgery. 2020;86(3):391-399.

12. Lima GLO, Dezamis E, Corns R, et al. Surgical resection of incidental diffuse gliomas involving eloquent brain areas. Rationale, functional, epileptological and oncological outcomes. Neurochirurgie. 2017;63(3):250-258.

13. Duffau H. Awake surgery for incidental WHO grade II gliomas involving eloquent areas. Acta Neurochir (Wien). 2012; 154(4):575-584.

14. Gogos AJ, Young JS, Morshed RA, et al. Awake glioma surgery: technical evolution and nuances. J Neurooncol. 2020; 147(3):515-524.

15. Hervey-Jumper SL, Li J, Lau D, et al. Awake craniotomy to maximize glioma resection: methods and technical nuances over a 27-year period. J Neurosurg. 2015;123(2):325-339.

16. Han SJ, Morshed RA, Troncon I, et al. Subcortical stimulation mapping of descending motor pathways for perirolandic gliomas: assessment of morbidity and functional outcome in 702 cases. J Neurosurg. 2018;131(1):201-208.

17. Shah AH, Madhavan K, Sastry A, Komotar RJ. Managing intracranial incidental findings suggestive of low-grade glioma: learning from experience. World Neurosurg. 2013;80(5): e75-e77.

18. Mandonnet E, de Witt Hamer P, Pallud J, et al. Silent diffuse low-grade glioma: toward screening and preventive treatment? Cancer. 2014;120(12):1758-1762.

19. Wijnenga MMJ, Mattni T, French PJ, et al. Does early resection of presumed low-grade glioma improve survival? A clinical perspective. J Neurooncol. 2017;133(1):137-146.

20. O'Connor M, Ryan J, Foley S. A review of cross-sectional imaging, ultrasound and nuclear medicine utilization patterns in paediatric patients in Ireland, 2003-12. Br J Radiol. 2015; 88(1048):20140767.

21. Smith-Bindman R, Miglioretti DL, Larson EB. Rising use of diagnostic medical imaging in a large integrated health system. Health Aff (Millwood). 2008;27(6):1491-1502.
22. Ciarrapico AM, Ugenti R, Di Minco L, et al. Diagnostic imaging and spending review: extreme problems call for extreme measures. Radiol Med (Torino). 2017;122(4):288-293.

23. Smith-Bindman R, Kwan ML, Marlow EC, et al. Trends in use of medical imaging in US health care systems and in Ontario, Canada, 2000-2016. JAMA. 2019;322(9):843-856.

24. Murphy ES, Leyrer CM, Parsons M, et al. Risk factors for malignant transformation of low-grade glioma. Int J Radiat Oncol Biol Phys. 2018;100(4):965-971.

25. Geurts M, van den Bent MJ. On high-risk, low-grade glioma: what distinguishes high from low? Cancer. 2019;125(2): 174-176.

26. Duffau H, Lopes M, Arthuis F, et al. Contribution of intraoperative electrical stimulations in surgery of low grade gliomas: a comparative study between two series without (1985-96) and with (1996-2003) functional mapping in the same institution. J Neurol Neurosurg Psychiatry. 2005;76(6): $845-851$.

27. Majchrzak K, Kaspera W, Bobek-Billewicz B, et al. The assessment of prognostic factors in surgical treatment of lowgrade gliomas: a prospective study. Clin Neurol Neurosurg. 2012;114(8):1135-1144.

28. McGirt MJ, Chaichana KL, Gathinji M, et al. Independent association of extent of resection with survival in patients with malignant brain astrocytoma. J Neurosurg. 2009;110(1): $156-162$.

29. Trinh VT, Davies JM, Berger MS. Surgery for primary supratentorial brain tumors in the United States, 2000-2009: effect of provider and hospital caseload on complication rates. J Neurosurg. 2015;122(2):280-296.

30. Cochereau J, Herbet G, Duffau H. Patients with incidental WHO grade II glioma frequently suffer from neuropsychological disturbances. Acta Neurochir (Wien). 2016;158(2): 305-312.

31. Venkatesh HS, Tam LT, Woo PJ, et al. Targeting neuronal activity-regulated neuroligin-3 dependency in high-grade glioma. Nature. 2017;549(7673):533-537.

32. Venkatesh HS, Morishita W, Geraghty AC, et al. Electrical and synaptic integration of glioma into neural circuits. $\mathrm{Na}$ ture. 2019;573(7775):539-545.

\section{Disclosures}

The authors report no conflict of interest concerning the materials or methods used in this study or the findings specified in this paper.

\section{Author Contributions}

Conception and design: Gogos, Young, Morshed, Potts, HerveyJumper, Berger. Acquisition of data: Gogos, Young, Pereira, Potts. Analysis and interpretation of data: Gogos, Young, Pereira, Morshed, Hervey-Jumper. Drafting the article: Gogos, Young, Pereira, Morshed. Critically revising the article: all authors. Reviewed submitted version of manuscript: all authors. Approved the final version of the manuscript on behalf of all authors: Gogos. Statistical analysis: Gogos. Study supervision: HerveyJumper, Berger.

\section{Correspondence}

Andrew J. Gogos: St. Vincent's Hospital, Melbourne, Victoria, Australia. andrew.gogos2@svha.org.au. 